\title{
In-silico identification of phenotype-biased functional modules
}

\author{
Kanchana Padmanabhan ${ }^{1,2}$, Kevin Wilson ${ }^{5}$, Andrea M Rocha ${ }^{3}$, Kuangyu Wang ${ }^{4}$, James R Mihelcic ${ }^{3}$, \\ Nagiza F Samatova ${ }^{1,2^{*}}$ \\ From IEEE International Conference on Bioinformatics and Biomedicine 2011 \\ Atlanta, GA, USA. 12-15 November 2011
}

\begin{abstract}
Background: Phenotypes exhibited by microorganisms can be useful for several purposes, e.g., ethanol as an alternate fuel. Sometimes, the target phenotype maybe required in combination with other phenotypes, in order to be useful, for e.g., an industrial process may require that the organism survive in an anaerobic, alcohol rich environment and be able to feed on both hexose and pentose sugars to produce ethanol. This combination of traits may not be available in any existing organism or if they do exist, the mechanisms involved in the phenotype-expression may not be efficient enough to be useful. Thus, it may be required to genetically modify microorganisms. However, before any genetic modification can take place, it is important to identify the underlying cellular subsystems responsible for the expression of the target phenotype.
\end{abstract}

Results: In this paper, we develop a method to identify statistically significant and phenotypically-biased functional modules. The method can compare the organismal network information from hundreds of phenotype expressing and phenotype non-expressing organisms to identify cellular subsystems that are more prone to occur in phenotype-expressing organisms than in phenotype non-expressing organisms. We have provided literature evidence that the phenotype-biased modules identified for phenotypes such as hydrogen production (dark and light fermentation), respiration, gram-positive, gram-negative and motility, are indeed phenotype-related.

Conclusion: Thus we have proposed a methodology to identify phenotype-biased cellular subsystems. We have shown the effectiveness of our methodology by applying it to several target phenotypes. The code and all supplemental files can be downloaded from (http://freescience.org/cs/phenotype-biased-biclusters/).

\section{Background}

Phenotypes that certain microorganisms express includes breaking down the lignocellulosic barrier of biomass, biodegradation of various environmental contaminants etc. Tackling problems in the areas of bioremediation and bioenergy with the help of genetic engineering requires as a first step identifying the cellular subsystems that are involved in the phenotype-expression by an organism. The phenotype-related cellular subsystems may be detected using laboratory

\footnotetext{
* Correspondence: samatova@csc.ncsu.edu

'Department of Computer Science, North Carolina State University, Raleigh, 27695, USA

Full list of author information is available at the end of the article
}

experimentation. However, to supplement experimentation methods, computational methodologies need to be used.

Biological relationships (e.g., protein functional associations) between proteins are often modeled as networks (functional association networks from STRING [1]), where each node is a protein and every pair of functionally associated proteins is connected with an edge. Functional association between proteins is derived from a number of clues like experimental data, genefusion, co-occurrence of the corresponding genes on the same operon, etc. The subgraphs of these networks can model the cellular subsystems.

Evolutionary conservation of cellular subsystems can be used as one clue to identify the phenotype-related 
cellular subsystems [2]. The cellular systems associated with a phenotype are more likely to be present across phenotype-expressing organisms and are less likely to be present across phenotype non-expressing organisms [2]. This strategy can be utilized to identify cellular subsystems that are likely phenotype-related.

An earlier work by Schmidt et al [2] focused on identifying phenotype-related functional modules that were modeled as cliques. Functional modules that have a clique structure require that every pair of proteins in the module has an edge between them. The density of the subgraph modeled as a clique is 1 . Density is the ratio of the number of edges in the subgraph to the total number of possible edges in the subgraph. This method was one of the first to identify phenotype-related subgraphs. However, their subgraph identification condition is too stringent to model all biological functional modules. This is primarily because biological networks are prone to missing information (like missing edges) [3]. Paccanarot et al [4] explain that most of the errors in the networks are false-negatives, i.e., edges that were not predicted. Hence, Schmidt et al [2] method may not identify the complete phenotype-related cellular subsystems. They acknowledge this as a drawback and use the identified cliques as input into another algorithm called DENSE [5], that can extract extended subsystems from a single organismal network. These subsystems may or may not be related towards the target phenotype.

Additionally, Schmidt et al [2] method requires two inputs: the parameter $\alpha$-the least number of phenotypeexpressing organisms the identified clique has to be present in and the parameter $\beta$-the number of phenotype non-expressing organisms the identified clique can be present in. These parameters may be hard to estimate beforehand and, hence, multiple runs with different parameter values may be required.

Spirin et al [6] showed that significantly dense "nonclique" clusters formed biologically relevant functional modules. They provide an example of a functional module associated with cell-cycle regulation consisting of cyclins (CLB1-4 and CLN2), cyclin-dependent kinases (CKS1 and CDC28), and a nuclear import protein NIP29 identified from Saccharomyces cerevisiae network that is not a clique. Hwang et al [7] showed that maximal clique enumeration methods discard over $90 \%$ of network nodes when applied to the PPI network of Saccharomyces cerevisiae. Hendrix et al [5] identified "non-clique" functional modules that were verified by literature. Habibi et al [8] showed that protein complexes that are usually thought to be cliques could also have different topologies (MIPS ID: 510.40.10 and 550.1.213 complexes) and this could primarily be due to the fact that biological data sources contain noise and possibly do not contain the entire information due to limitations of experiments. Additionally, their detailed study of the densities of the existing protein complexes from various sources [9-13] has revealed that many complexes have density less than 0.1 .

In this paper, we propose a methodology (Figure 1 and Figure 2) to identify the statistically significant functional modules that are phenotype-biased. Phenotypebiased means that it is more conserved across phenotype-expressing organisms and less conserved across phenotype non-expressing organisms. The functional modules are identified by a comparative analysis using both phenotype-expressing and phenotype non-expressing organisms. The structure of the functional modules is a subgraph that is a connected component which is then filtered to identify the statistically significant components. The method does not require parameters similar to the parameters $\alpha$ and $\beta$, in [2] to decide the number of organisms the resulting subsystem should be present in.

In our earlier conference paper [14], we identified the biclusters common to a set of phenotype expressing organisms and analyzed these biclusters for potential cross-talking pathways. In this paper, we extend the methodology to utilize phenotype non-expressing organismal information to identify phenotype-biased functional modules. This increases the confidence in the phenotype-relatedness of the identified modules. The phenotype-bias is quantified using the hypergeometric statistical test. In [14], we performed the analysis only for the hydrogen production phenotype. Here, we have also analyzed respiration, gram-positivity, gram-negativity and motility phenotypes.

\section{Results}

\section{Experimental setup}

We set up experiments with four different phenotypes, hydrogen production (dark and light fermentation), respiration, gram-stain, and motility. The organisms for each phenotype were identified using literature search $[15,16]$. The functional association network for each organism was obtained from the STRING [1] database and the edge score cutoff used was 700 (termed as high confidence [1]).

\section{Hydrogen production}

Biological hydrogen production is being looked at as a source of alternate energy and there are plenty of microorganisms that can utilize different organic substrates to produce hydrogen. This makes it a useful alternate energy option to explore [17-19]. Identifying cellular subsystems related to hydrogen production will be extremely useful to genetic engineers looking to make the process of biological hydrogen production more efficient. The light and dark fermentation are two 


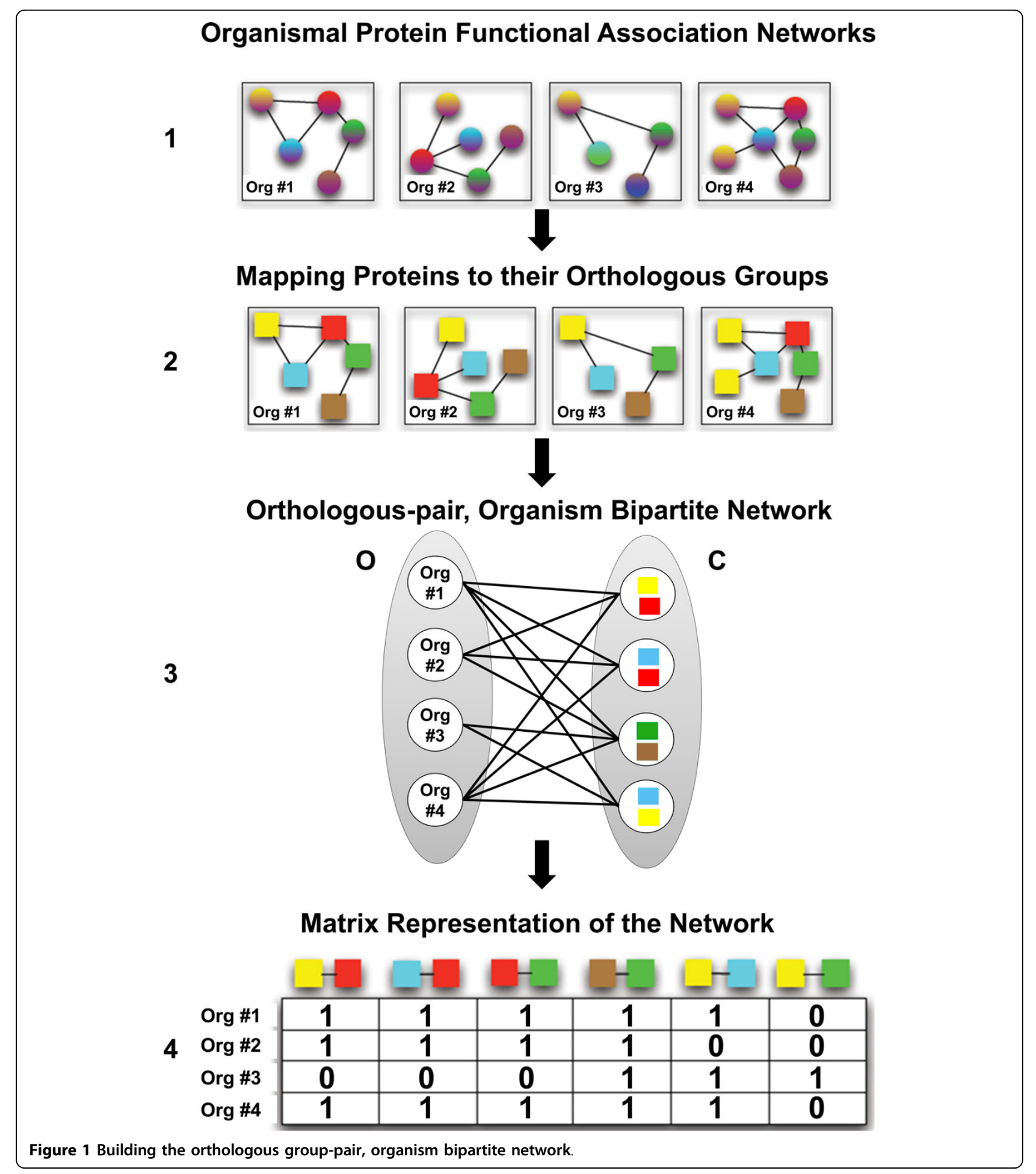

important sub-phenotypes of hydrogen production and experiments based on these phenotypes have been discussed in this section.

\section{Light fermentation}

Initial review of the light fermentation clusters shows the presence of a set of 13 identical COGs found across all 8 COG clusters. These "core" COGs include genes necessary for synthesis of hydrogenase complex(es).

Nitrogen-fixation is the process, in which nitrogenase catalyzes the conversion of nitrogen gas to ammonia and inadvertently results in the production of hydrogen gas as a byproduct [20,21]. Two COGs (COG2710 and 


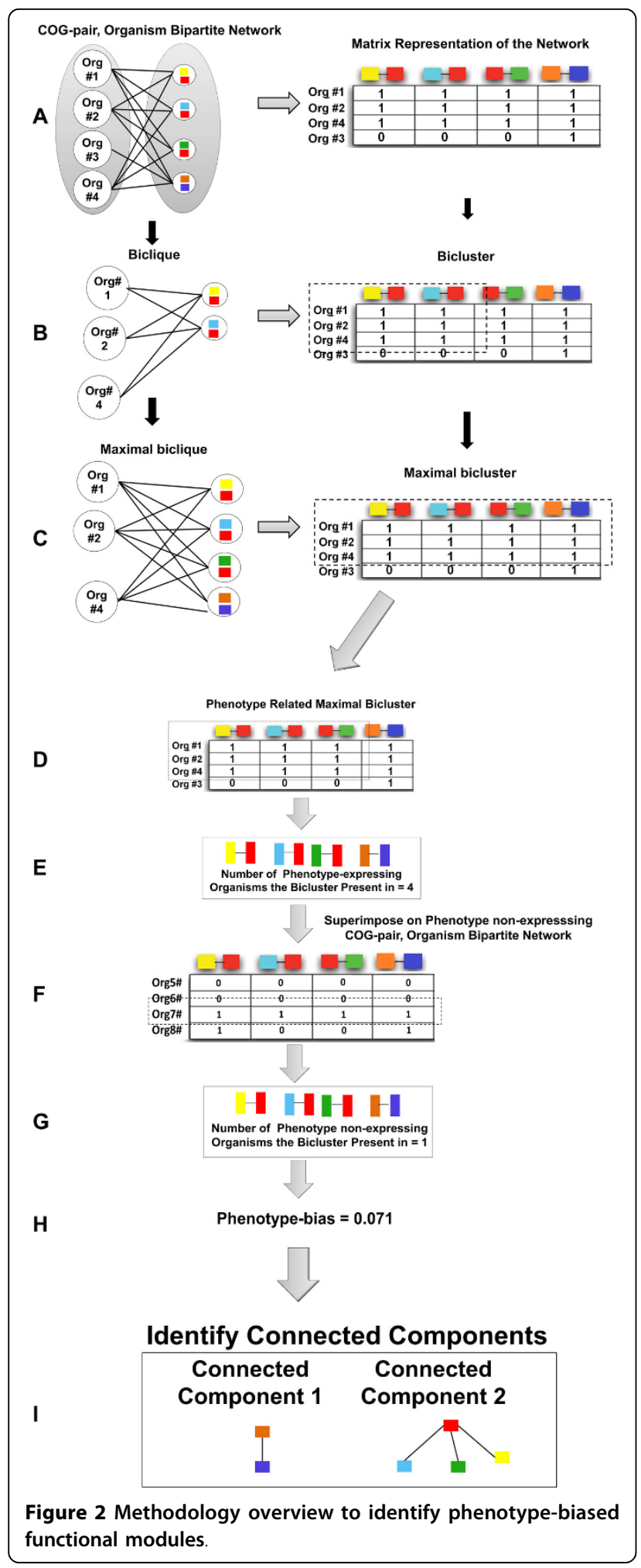

COG1348), which are associated with the expression of two key proteins, nitrogenase iron protein $(\mathrm{NifH})$ and molybdenum iron protein [20], were present across all the clusters. Although, the presence of these two proteins is essential for nitrogen-fixation to be carried out by light fermenting microorganisms, expression of various genes in other metabolic pathways plays important roles in either directly or indirectly regulating the expression of genes encoding NifH proteins. These proteins include ferric iron regulation proteins (sigK, clpB, and fur-related), ammonia ligase (glnA), and nitrogenase [22]. In this study, glutamate ammonia ligase $(g \ln \mathrm{A})$, a key gene for nitrogenase (NifH), and genes encoding proteins for iron uptake, are assembled in the same cluster. In Anabaena, iron uptake proteins and some nitrogen proteins (e.g., Ntc) have been shown to regulate genes encoding glutamate synthetase $(g \ln \mathrm{A})$ [23]. Review of the role of glutamine synthetase in Anabaena indicates that this enzyme is responsible for regulating nitrogenase activity, thus impacting hydrogen production [23]. The indirect regulation of nitrogenase by iron uptake proteins provides an example of cross-talk between iron and nitrogen-related metabolic pathways.

In addition to nitrogenase, proteins associated with the synthesis of uptake or expression of hydrogenase, were identified in 11 of the 19 COGs present in Table 1. Hydrogen uptake proteins help with removing excess hydrogen to maintain the reducing environment in cells [24]. We also identified a number of proteins (e.g., Hyd and Hyp) involved in formation of [NiFe]-uptake hydrogenases. The presence of maturation hydrogenase factors (COG0068, COG0298, COG0309) and accessory proteins for uptake of nickel (COG0378) are consistent with literature reports describing the structure of hydrogenase complexes. Inclusion of hy-drogenase proteins in Table 1 is likely due to the relationship of hydrogenase proteins with iron uptake genes. To function properly, iron is needed to form the NiFe center present in the large hydrogenase subunit (HupL) [25]. As such, hydrogenase maturation is dependent on cross-talks with iron uptake.

In previous studies by Lopez-Gollomon [23], the nitrogen regulator protein NtcA was found to work together with the iron-uptake protein, Fur, to co-regulate genes involved in various metabolic functions. Metabolic functions co-regulated include the transcriptional regulation protein and glutamine synthesis [26]. In this study, genes encoding iron uptake regulator proteins (COG0735) were clustered together with genes encoding glutamine synthetase (COG0174). The coappearance of these two COGs suggests the possible cross-talk between iron uptake and ammonia assimilation networks. In addition, there is indication that hydrogenase proteins, such as HupUV, are involved in regulating the glutamine synthetase gene, glnAII, in some organisms $[20,27]$.

\section{Dark fermentation}

Unlike light fermentation, we did not observe a large set of COGs present across all clusters. For this set of 


\begin{tabular}{|c|c|}
\hline COG ID & COG Description \\
\hline COG0068 & Hydrogenase maturation factor \\
\hline COG0298 & Hydrogenase maturation factor \\
\hline COG0309 & Hydrogenase maturation factor \\
\hline COG0374 & Ni,Fe-hydrogenase I large subunit \\
\hline COG0375 & $\begin{array}{l}\text { Zn finger protein HypA/HybF(possibly regulating } \\
\text { hydrogenase expression) }\end{array}$ \\
\hline COG0378 & $\begin{array}{l}\text { Ni2+-binding GTPase involved in regulationof expression and } \\
\text { maturation of urease and hydrogenase } 1\end{array}$ \\
\hline COG0409 & Hydrogenase maturation factor \\
\hline COG0680 & $\mathrm{Ni}$, Fe-hydrogenase maturation factor \\
\hline COG1740 & Ni,Fe-hydrogenase I small subunit \\
\hline COG0174 & Glutamine synthetase \\
\hline COG0535 & Predicted Fe-S oxidoreductases \\
\hline COG0716 & Flavodoxins \\
\hline COG1348 & Nitrogenase subunit NifH (ATPase) \\
\hline COG2082 & Precorrin isomerase \\
\hline $\operatorname{cog} 2710$ & Nitrogenase molybdenum-iron protein,alpha and beta chains \\
\hline COG2370 & Hydrogenase/urease accessory protein \\
\hline COG1941 & Coenzyme F420-reducing hydrogenase, gamma subunit \\
\hline COG3259 & Coenzyme F420-reducing hydrogenase, alpha subunit \\
\hline COG0735 & $\mathrm{Fe} 2+\mathrm{Zn} 2+$ uptake regulation proteins \\
\hline
\end{tabular}

organisms, only two COGs were identified as present across all clusters. This may be partially due to the following two reasons. First, the selection of species and species diversity have some impact on the types of clusters generated. Second, dark fermentation organisms tend to utilize a greater variety of fermentation pathways, such as acetate fermentation and butyrate fermentation pathways [28]. Greater variation in fermentation routes will not produce as large of a "core" set of COGs across all clusters.

An example of COG clusters identified in dark fermentative bacteria is present in Table 2. In this cluster, 13 different COGs consisting of proteins that are either directly or indirectly responsible for the uptake or production of hydrogen, are present. Of these COGs, 7 are related to the synthesis or expression of [NiFe]-hydrogenase, an enzyme that catalyses the reversible oxidation of molecular hydrogen, and plays a vital role in anaerobic metabolism [27]; the others are involved in nitrogen and iron metabolic pathways that include proteins like nitrogenase, iron uptake proteins, such as Fur (COG0735), ammonia assimilation proteins, such as glutamine synthetase (COG3968), and proteins involved in electron transfer. Previous findings by Butland et al. [29] show that the presence of proteins (e.g., HypE, HypD, HupS, HupD) is typically associated with hydrogen uptake $[25,30]$. Based on the other genes (e.g., hybG, hupS) present in the cluster, we can predict that

\begin{tabular}{ll} 
Table 2 COGs associated with dark fermentation \\
identified by the method \\
\hline COG ID & COG Description \\
\hline COG0298 & Hydrogenase maturation factor \\
COG0309 Hydrogenase maturation factor \\
COG0374 Ni,Fe-hydrogenase I large subunit \\
COG0409 Hydrogenase maturation factor \\
COG0680 Ni,Fe-hydrogenase maturation factor \\
COG1740 Ni,Fe-hydrogenase I small subunit \\
COG0535 Predicted Fe-S oxidoreductases \\
COG1348 Nitrogenase subunit NifH (ATPase) \\
COG2710 Nitrogenase molybdenum-iron protein, alpha and beta \\
chains \\
COG0716 Flavodoxins \\
COG0735 Fe2+/Zn2+ uptake regulation proteins \\
COG2082 & Precorrin isomerase \\
COG3968 & Uncharacterized protein related to glutamine synthetase
\end{tabular}

[NiFe]-hydrogenase is associated with hydrogen uptake in this group of organisms.

In addition to hydrogenase maturation and expression proteins, Fe-S oxidoreductases were identified. As part of the structure of [NiFe]-hydrogenase, Fe-S metal centers are located on the small subunit of the hydrogenase complex [25,27]. Thus, it is expected that iron uptake pathway would cross-talk with hydrogenase-related pathways. Furthermore, the iron uptake pathway also cross-talks with nitrogen metabolism, in a sense that iron uptake proteins can be involved indirectly in nitrogen metabolism through regulation of nitrogenase and maintaining the reducing environment in the cell through hydrogen uptake (hydrogenase) [26,31].

It has been shown that cross-talk between iron uptake and nitrogen metabolism enables regulation of ammonia assimilation [21]; it may be possible that the uncharacterized glutamine synthetase protein in Table 2 is subject to such regulation. In our results, the gene encoding the uncharacterized glutamine synthetase proteins was only present in a few species, including Clostridium acetobutylicum and Clostridium beijerinckii, which both contained nitrogenase and hydrogenase enzymes. It has been demonstrated that, in light fermenting organisms, such as Rhodopseudomonas palustris, glutamine synthetase is regulated by hydrogenase accessory proteins (HupUV) [21]. However, to the best of our knowledge, this relationship has not been described in dark fermentation organisms. This knowledge increases the probability that the uncharacterized glutamine synthetase protein maybe present in the COG cluster oweing to its association with nitrogenase proteins, which may further indicate a possible cross-talk between ammonia assimilation and nitrogen metabolism. 


\section{Motility}

The motility experiment was set up with a set of 85 motile and 56 non-motile organisms chosen from Slonim et al [16]. The method identified clusters that contained COG1360, COG1558, COG1157, COG1684, and COG1536 (Table 3). All these COGS are related to flagella proteins. The flagella proteins are those that enable the organisms to move. The method also found COG0643, COG0835, and COG0784 that are related to bacterial chemotaxis. It is well known that chemotaxis controls an organism's movement with respect to the chemical composition of its environment. For example, it helps the organism moves to the areas where there is very high concentration of food [32].

COGS related to the Type III secretion system (COG1766, COG1684, COG1987, COG1338, and COG1886) were also identified. It has been shown that Type III secretion proteins share similarities with flagella proteins in structure and function [33]. Additionally, we identified COG0835, COG0643, COG1344, COG1291, COG0784, COG1508, and COG1191 associated with the two-component systems. This is a signaling pathway that regulates motility [34].

\begin{tabular}{|c|c|}
\hline COG ID & COG Description \\
\hline COG1843 & Flagellar hook capping protein \\
\hline COG1291 & Flagellar motor component \\
\hline COG1344 & Flagellin and related hook-associated proteins \\
\hline COG1256 & Flagellar hook-associated protein \\
\hline COG1338 & Flagellar biosynthesis pathway, component FliP \\
\hline COG4786 & Flagellar basal body rod protein \\
\hline COG1360 & Flagellar motor protein \\
\hline COG1558 & Flagellar basal body rod protein \\
\hline COG1157 & Flagellar biosynthesis,type III secretory pathway ATPase \\
\hline COG1684 & Flagellar biosynthesis pathway, component FliR \\
\hline COG1536 & Flagellar motor switch protein \\
\hline COG1766 & Flagellar biosynthesis/type III secretory pathway lipoprotein \\
\hline COG1684 & Flagellar biosynthesis pathway,component FliR \\
\hline COG1987 & Flagellar biosynthesis pathway,component FliQ \\
\hline COG1338 & Flagellar biosynthesis pathway, component FliP \\
\hline COG1886 & Flagellar motor switch/type III secretory pathway protein \\
\hline COG0643 & Chemotaxis protein histidine kinaseand related kinases \\
\hline COG0835 & Chemotaxis signal transduction protein \\
\hline COG0784 & FOG: CheY-like receiver \\
\hline COG0643 & Chemotaxis protein histidine kinase and related kinases \\
\hline COG1508 & $\begin{array}{l}\text { DNA-directed RNA polymerase specialized sigma subunit, } \\
\text { sigma54 homolog }\end{array}$ \\
\hline COG1191 & DNA-directed RNA polymerase specialized sigma subunit \\
\hline
\end{tabular}

\section{Respiration}

This experiment was set up with a set of 77 aerobic organisms and 57 anaerobic organisms. For aerobic respiration, COGs related to the enzymes present in the TCA cycle were identified. They are COGs related to citrate synthase (COG0372), acitonase (COG1048), and Malate dehydrogenases (COG0039) (Table 4). Some COGs such as the malate synthase (COG2225), isocitrate synthase (COG2224), glyoxylate bypass were also found. The entire list of TCA-related COGs identified can be found in Table 4. There were also other literature verified COGs (COG0843, COG0109,COG1048, COG1622, COG1845, and COG0372) found by the method described in [35].

For anaerobic experiment, we found COG1924, COG1592, COG2221, and COG2033. The COG1924 is related to oxygen sensitive proteins [36] (Table 5). The other COGs were pulled out computationally by another genotype-phenotype methods [35,36] applied to the anaerobic phenotype. We also identified COGs from the Arginine and proline metabolism, the reason for this could be attributed to the L-argnine which could serve as an energy source for anaerobes.

\section{Gram-positive and gram-negative}

This experiment was set up with a set of 61 gram positive bacteria and 109 gram negative bacteria. For gram negativity, COG2877, COG2885, COG1044, COG1519, COG0763, and others related to the Lipopolysaccha-ride biosynthesis were found (Table 6). This pathway has been shown to be related to gram-negativity [33]. Another set consisting of COG0043, COG0163, COG2227, COG1008, and COG1005 were found. These are associated with the ubiquinone pathway that is also shown to be associated with gram-negativity [33]. The

\begin{tabular}{l} 
Table 4 COGs associated with aerobic respiration \\
identified by the method \\
\hline COG ID COG Description \\
\hline COG0372 Citrate synthase \\
COG1048 Aconitase A \\
COG0045 Succinyl-CoA synthetase, beta subunit \\
COG0074 Succinyl-CoA synthetase, alpha subunit \\
COG0479 $\begin{array}{l}\text { Succinate dehydrogenase/fumarate reductase,Fe-S protein } \\
\text { subunit }\end{array}$ \\
COG1053 Succinate dehydrogenase/fumarate reductase,flavoprotein \\
subunit \\
COG2142 Succinate dehydrogenase,hydrophobic anchor subunit \\
COG0039 Malate/lactate dehydrogenases \\
COG2224 Isocitrate lyase \\
COG2225 Malate synthase \\
COG2084 $\begin{array}{l}\text { 3-hydroxyisobutyrate dehydrogenaseand related beta- } \\
\text { hydroxyacid dehydrogenases }\end{array}$ \\
COG2379 Putative glycerate kinase
\end{tabular}


Table 5 COGs associated with anaerobic respiration identified by the method

\begin{tabular}{ll}
\hline COG ID & COG Description \\
\hline COG1924 & $\begin{array}{l}\text { Activator of 2-hydroxyglutaryl-CoA dehydratase(HSP70-class } \\
\text { ATPase domain) }\end{array}$ \\
COG1592 & Rubrerythrin \\
COG2221 & $\begin{array}{l}\text { Dissimilatory sulfite reductase(desulfoviridin), alpha and beta } \\
\text { subunits }\end{array}$ \\
COG2033 Desulfoferrodoxin
\end{tabular}

COG0848 found by the method has been shown to be associated with the target phenotype [37].

From gram-positive bacteria (Table 7), the method identified COG3764 and COG3773. COG3674 relates to plasma membrane proteins and was identified by previous research as related to gram-positivity [37]. The COG3773 is associated with endospore formation that usually occurs in gram-positive bacteria when there is a

Table 6 COGs associated with gram negativity identified by the method

\begin{tabular}{|c|c|}
\hline COG ID & COG Description \\
\hline COG2877 & $\begin{array}{l}\text { 3-deoxy-D-manno-octulosonic acid(KDO) 8-phosphate } \\
\text { synthase }\end{array}$ \\
\hline COG2885 & $\begin{array}{l}\text { Outer membrane protein andrelated peptidoglycan- } \\
\text { associated (lipo)proteins }\end{array}$ \\
\hline COG1044 & UDP-3-O-[3-hydroxymyristoyl]glucosamine $\mathrm{N}$-acyltransferase \\
\hline COG1519 & 3-deoxy-D-manno-octulosonic-acid transferase \\
\hline COG0763 & Lipid A disaccharide synthetase \\
\hline COG0838 & NADH:ubiquinone oxidoreductase subunit 3 (chain A) \\
\hline COG0337 & 3-dehydroquinate synthetase \\
\hline COG0852 & NADH:ubiquinone oxidoreductase 27 kD subunit \\
\hline COG1143 & $\begin{array}{l}\text { Formate hydrogenlyase subunit 6/NADH:ubiquinone } \\
\text { oxidoreductase } 23 \mathrm{kD} \text { subunit (chain I) }\end{array}$ \\
\hline COG0713 & NADH:ubiquinone oxidoreductase subunit 11 or $4 \mathrm{~L}$ (chain $\mathrm{K}$ ) \\
\hline COG0649 & NADH:ubiquinone oxidoreductase 49 kD subunit 7 \\
\hline COG0382 & $\begin{array}{l}\text { 4-hydroxybenzoate polyprenyltransferaseand related } \\
\text { prenyltransferases }\end{array}$ \\
\hline COG0043 & $\begin{array}{l}\text { 3-polyprenyl-4-hydroxybenzoate decarboxylaseand related } \\
\text { decarboxylases }\end{array}$ \\
\hline COG0163 & 3-polyprenyl-4-hydroxybenzoate decarboxylase \\
\hline COG2227 & $\begin{array}{l}\text { 2-polyprenyl-3-methyl-5-hydroxy-6-metoxy-1,4-benzoquinol } \\
\text { methylase }\end{array}$ \\
\hline COG1008 & NADH:ubiquinone oxidoreductase subunit 4 (chain M) \\
\hline COG1005 & NADH:ubiquinone oxidoreductase subunit 1 (chain H) \\
\hline COG1663 & Tetraacyldisaccharide-1-P 4'-kinase \\
\hline COG0774 & UDP-3-O-acyl-N-acetylglucosamine deacetylase \\
\hline COG1212 & CMP-2-keto-3-deoxyoctulosonic acid synthetase \\
\hline COG0859 & ADP-heptose:LPS heptosyltransferase \\
\hline COG2908 & Uncharacterized protein conserved in bacteria \\
\hline COG2870 & $\begin{array}{l}\text { ADP-heptose synthase, bifunctionalsugar kinase/ } \\
\text { adenylyltransferase }\end{array}$ \\
\hline COG3307 & Lipid A core - O-antigen ligase and related enzymes \\
\hline COG0445 & NAD/FAD-utilizing enzymeapparently involved in cell division \\
\hline COG0848 & Biopolymer transport protein \\
\hline
\end{tabular}

Table 7 COGs associated with gram positivity identified by the method

\begin{tabular}{ll}
\hline COG ID & COG Description \\
\hline COG3764 & Sortase \\
COG3773 & Cell wall hydrolyses involved in spore germination \\
COG0619 & ABC-type cobalt transport system, permease component \\
CbiQ and related transporters
\end{tabular}

lack of nutrients. We also found COG0619 and COG1122 as single connected component; these COGs are associated with uptake of MET/or MET-precursors, which are associated with regulation of genes involved in amino acid metabolism in gram-positive bacteria [38].

\section{Discussion}

In summary, the proposed methodology identifies the phenotype-biased cellular subsystems. The results of the method provide clues on which subsystems are co-present to help in phenotype expression. This information could potentially be put to use by genetic engineers. However, there are three points that should be pondered over.

There are several points that are worth bringing out especially when analyzing network data of both phenotype-expressing and phenotype non-expressing organisms. The first point is on the importance of phylogenetic diversity of the underlying organisms. Our results depend on the organisms chosen for the experiment. There could be cases where the conserved biclusters were identified purely due to the fact that the chosen organisms were phylogentically close. Thus, as part of future work we will look into incorporating some scoring mechanism, such that the identified phenotype-biased biclusters are also conserved across a set of phylogenetically diverse set of organisms.

The second point of discussion stems from the fact the same organism can express multiple phenotypes. Currently we look at only one phenotype at a time. The current methods that analyze multiple phenotypes at the same time [33] do so by looking at one phenotype at a time and by correlating the results of the different experiments. Analyzing multiple (possibly related) phenotypes together might provide new insights into pathway cross-talking mechanisms. Another direction of future work is to extend our method to work with multiple phenotypes.

The third point of discussion stems from the fact that a phenotype may have several subphenotypes. For example, all hydrogen producing organisms do not express the phenotype in the same manner. Hydrogen production has three subphenotypes dark fermentation, light fermentation and biophotolysis, two of those were 
discussed in this paper. Thus, when methods seek to identify phenotype-related systems using multiple organism data, it is imperative to identify systems present in any subset of organisms as opposed to all the organisms. The subsystem present in a subset (as opposed to all) may be a specific path (subphenotypes) to carry out the phenotype. Our methodology models that intuition by using the bicluster definition for the functional module, thus naturally allowing the identified cluster to be present in any organism subset (of size $\geq 2$ ).

\section{Conclusion}

We have developed a method to identify phenotypebiased functional modules by utilizing both phenotypeexpressing and phenotype non-expressing organismal network data. By applying our method to four phenotypes, hydrogen production, gram stain, motility and respiration, we were able to identify functional modules that were associated with the target phenotypes. The findings were validated via literature evidence.

\section{Methods}

\section{Orthologous group-pair, organism bipartite network}

In order to identify functional modules across a given set of organismal protein functional association networks, we need a representation that would help us enumerate these modules efficiently. The organismal protein functional association network is obtained from STRING database [39], each node is a protein and a pair of proteins are connected by an edge if there is some evidence of their functional association. Some examples of the evidences considered in STRING are gene fusion, co-occurrence on the same operon, coexpression etc. In this paper we propose the orthologous group-pair, organism bipartite network that combines the information present in all of the individual organismal protein functional association networks into one single network (Figure 1).

As a first step to constructing this network, we need some kind of transformation that would help us understand the commonalty and differences among the networks. One such transformation is replacing all proteins in all of the organismal networks with their corresponding orthologous group IDs (Figure 1.2). The most common representation used in biology is the manually curated Clusters of Orthologous Groups (COGs) [40].

In the second step, we construct two sets, $O$ and $C$ (Figure 1.3). In $C$, each element is a pair $(x, y)$, where both $x$ and $y$ are COGs. In $O$, each element represents an organism. These two sets become the two partites of the graph.

As a final step, we construct the orthologous grouppair, organism bipartite network (Figure 1.3), $N=(O, C$, $E)$. An edge $(a, b) \in E$, where $a \in O$ and $b=(u, v) \in C$ exists if and only if the COG pair $(u, v)$ is functionally associated in organism $a$, i.e., in the organismal protein functional association network $A=(V(A), E(A))$ corresponding to organism $a, \exists x, y \in V(A): x$ and $y$ belong to orthologous cluster groups $u$ and $v$, respectively, and $(x, y) \in E(A)$. Since in this paper we make use of COGs, the network $N$ will henceforth be referred to as the COG-pair, organism bipartite network.

\section{Network representation and preprocessing}

The COG-pair, organism bipartite network, $N$ is represented using an adjacency matrix for the purpose of identifying the conserved functional modules (Figure 1.4). The organisms are the row-headers and each column header is a COG-pair. A matrix cell has a 1, if the corresponding organism (row-header) and the COG pair (column-header) are connected by an edge in network $N$. This matrix is typically sparse.

\section{Obtaining the conserved COG clusters}

As a first step to identifying the modules, we identify sets of COG edges that are conserved across two or more organisms. These sets can be represented as bicliques (Figure 2.B) in the COG-pair, organism bipartite network. To avoid enumerating the same information more than once, we only enumerate the maximal bicliques (Figure 2.C).

Definition 0.1 Given a bipartite graph $N=(O, C, E)$, a subgraph $S=\left(O^{\prime}, C^{\prime}, E^{\prime}\right)$ of $N$ is a biclique if $\forall a \in O^{\prime}$ and $b \in C^{\prime},(a, b) \in E^{\prime}$.

Definition $0.2 \mathrm{~A}$ biclique $S$ of $N$ is also maximal if there is no supergraph $S$ of $S$ that forms a biclique in $N$.

The problem of identifying maximal bicliques using the binary matrix representation translates to identifying the maximal biclusters (Figure 2.B) in the matrix. Although any biclustering technique that works on binary matrices would suffice, we chose Prelic et al.'s Bimax biclustering algorithm [41]. There are two reasons for this choice: (1) Bimax performs on par with the best biclustering techniques [41], and (2) It has also been shown that Bimax is able to output all the optimal (maximal) biclusters in the given binary matrix [41]. The algorithm uses a divide-and-conquer approach to enumerate maximal bicliques (maximal biclusters) in the COG-pair, organism bipartite network (Figure 2.C).

\section{Comparative analysis using phenotype non-expressing organisms}

So far, we have hypothesized that biclusters conserved across 2 or more phenotype-expressing organisms are likely phenotype related. We strengthen the notion of phenotype-related to phenotype-biased by performing comparative analysis using a set of both phenotypeexpressing and phenotype non-expressing organisms. 
Phenotype-biased biclusters are likely to be conserved more across a set of phenotype expressing organisms and less across a set of phenotype non-expressing organisms.

Figure 2.D-G shows the steps in the comparative analysis pipeline. The analysis begins with a set of biclusters identified in Figure 2.C. The orthologous group-pair, organism bipartite network is built for the set of phenotype non-expressing organisms. This network is then converted into its matrix representation (phenotype non-expression matrix). Each phenotype-related bicluster, identified previously, is now analyzed in the context of the phenotype non-expression matrix. We seek to identify the number of phenotype non-expressing organisms the bicluster is conserved in. This information can be utilized to calculate the phenotype-bias of this bicluster.

The phenotype-bias is quantified by using the hypergeometric statistical test. Let $P$ be the total number of organisms (both phenotype-expressing and phenotype non-expressing). Let $S$ be the total number of phenotype expressing organisms. Let $X$ be total number of organisms (both phenotype-expressing and phenotype nonexpressing) the bicluster $B$ is present in. Let $Y$ be the number of phenotype-expressing organisms the bicluster $B$ is present in. The bias ( $p$-value) of the bicluster $B$ is calculated as follows:

$$
\operatorname{bias}(B)=\frac{\left(\begin{array}{l}
S \\
Y
\end{array}\right) *\left(\begin{array}{l}
P-S \\
X-Y
\end{array}\right)}{\left(\begin{array}{l}
P \\
X
\end{array}\right)}
$$

We apply a $p$-value cutoff of 0.05 to identify all the phenotype-biased biclusters.

\section{Enumerating the connected components}

Each phenotype-biased maximal bicluster identified in the previous section represents the set of COG-COG edges conserved across the set of phenotype-expressing organisms. However, we cannot consider the COGCOG edge set as a functional module as is. A functional module has to be a connected subgraph of an organismal network as opposed to a collection of edges. A connected component subgraph is one where there is path between every pair of nodes in the subgraph. However, there is no guarantee that all the COG-COG edges in the bicluster are connected. Thus, all the connected component subgraphs from the COG-COG edge set of each bicluster are enumerated (Figure 2.I).

\section{Assessing statistical significance}

The results of the previous section only guarantee that the subgraphs output are connected components but there is no clear indication whether the subgraphs could potentially represent functional modules or if their occurrence was purely random. One way to check this would be to compare the density of each component with the density that could be obtained at random for a subgraph with the same number of nodes.

The Monte Carlo method [42,43], a robust statistical significance method, is utilized to assess the significance. For every connected component $S=(V, E)$, we calculate the density $\beta(S)$. We randomly sample subsets of $|E|$ COGs each from the set of all possible COGs $M$. We estimate an empirical $p$-value as $R / W$, where $W$ is the total number of random subsets generated $(W \sim 1000)$ and $R$ is the number of random subsets that produce a test statistics $\beta()$ greater than or equal to that of $\beta(S)$. We then use a cutoff (say 0.05) to identify the statistically significant components.

\section{Acknowledgements}

This work was supported in part by the U.S. Department of Energy, Office of Science, the Office of Advanced Scientific Computing Research (ASCR) and the Office of Biological and Environmental Research (BER) and the U.S. National Science Foundation (Expeditions in Computing). The work by A.M.R was supported by the Delores Auzenne Fellowship and the Alfred P. Sloan Minority PhD Scholarship Program. Oak Ridge National Laboratory is

managed by UT-Battelle for the LLC U.S. D.O.E. under contract no. DEAC05000R22725.

This article has been published as part of Proteome Science Volume 10 Supplement 1, 2012: Selected articles from the IEEE International Conference on Bioinformatics and Biomedicine 2011: Proteome Science. The full contents of the supplement are available online at http://www.proteomesci. com/supplements/10/S1

\section{Author details}

'Department of Computer Science, North Carolina State University, Raleigh, 27695, USA. ${ }^{2}$ Computer Science and Mathematics Division, Oak Ridge National Laboratory, Oak Ridge, 37831, USA. ${ }^{3}$ Department of Civil and Environmental Engineering, University of South Florida, Tampa, 33620, USA. ${ }^{4}$ Bioinformatics Research Center, North Carolina State University, Raleigh, NC 27695, USA. ${ }^{5}$ RTI International, Durham, NC 27709, USA.

\section{Authors' contributions}

$\mathrm{KP}$ and $\mathrm{KW}$ developed and implemented the computational model and the algorithm and conducted computational experiments. $A R, K G$, and $K P$ provided biological validation. $\mathrm{KP}, \mathrm{KW}, \mathrm{KG}$, and $\mathrm{AR}$ provided the initial draft of the manuscript. JM suggested and supervised the study related to the hydrogen production from wastewater and waste materials. NS provided the problem statement, supervised the development of the computational methodology, and provided suggestions on methodology validation. JM and NF contributed to preparing the final version of the manuscript. All authors have read and approved the final manuscript.

\section{Competing interests}

The authors declare that they have no competing interests.

\section{Published: 21 June 2012}

\section{References}

1. Jensen L, Kuhn M, Stark M, Chaffron S, Creevey C, Muller J, Doerks T, Julien P, Roth A, Simonovic M, Bork P, von Mering C: STRING 8-a global view on proteins and their functional interactions in 630 organisms. Nucleic Acids Res 2009, 37:D412-D416.

2. Schmidt M, Rocha A, Padmanabhan K, Chen Z, Scott K, Mihelcic J, Samatova N: Efficient alpha, beta-motif finder for identification of phenotype-related functional modules. BMC Bioinformatics 2011, 12:440. 
3. Yan B, Gregory S: Finding missing edges and communities in incomplete networks. J Phys A 2011, 44:495102.

4. Paccanaro A, Trifonov V, Yu H, Gerstein M: Inferrng protein-protein interactions using interaction network topologies. Proceedings of the International Joint Conference on Neural Networks 2005.

5. Hendrix W, Rocha A, Padmanabhan K, Choudhary A, Scott K, Mihelcic J, Samatova N: DENSE: Efficient and prior knowledge-driven discovery of phenotype-associated protein functional modules. BMC Systems Biology 2011, 5:172.

6. Spirin V, Mirny L: Protein complexes and functional modules in molecular networks. Proc Natl Acad Sci 2003, 100:12123-12128.

7. Hwang W, Cho Y, Zhang A, Ramanathan M: A novel functional module detection algorithm for protein-protein interaction networks. Algorithms Mol Biol 2006, 1:24

8. Habibi M, Eslahchi C, Wong L: Protein complex prediction based on $\mathrm{k}$ connected subgraphs in protein interaction network. BMC Syst Biol 2010 4:129.

9. Stark C, Breitkreutz B, Reguly T, Boucher L, Breitkreutz A, Tyers M: BioGRID: A general repository for interaction datasets. Nucleic Acids Res 2006, 34: D535-D539.

10. Krogan N, Peng W, Cagney G, Robinson M, Haw R, Zhong G, Guo X, Zhang X, Canadien V, Richards D, Beattie B, Lalev A, Zhang W, Davierwala A, Mnaimneh S, Starostine A, Tikuisis A, Grigull J, Datta N, Bray J, Hughes T, Emili A, Greenblatt J: High-definition macromolecular composition of yeast RNA-processing complexes. Mol Cell 2004 13:225-239.

11. Ho Y, Gruhler A, Heilbut A, Bader G, Moore L, Adams S, Millar A, Taylor P, Bennett K, Boutilier K, Yang L, Wolting C, Donaldson I, Schandorff S, Shewnarane J, Vo M, Taggart J, Goudreault M, Muskat B, Alfarano C, Dewar D, Lin Z, Michalickova K, Willems A, Sassi H, Nielsen P, Rasmussen K, Andersen J, Johansen L, Hansen $L$, Jespersen $H$, Podtelejnikov A, Nielsen E, Crawford J, Poulsen V, Sorensen B, Matthiesen J, Hendrickson R, Gleeson F, Pawson T, Moran M, Durocher D, Mann M, Hogue C, Figeys D, Tyers M: Systematic identification of protein complexes in Saccharomyces cerevisiae by mass spectrometry. Nature 2002, 415:180-183.

12. Gavin A, Bosche M, Krause R, Grandi P, Marzioch M, Bauer A, Schultz J, Rick J, Michon A, Cruciat C, Remor M, Hofert C, Schelder M, Brajenovic M, Ruffner H, Merino A, Klein K, Hudak M, Dickson D, Rudi T, Gnau V, Bauch A, Bastuck S, Huhse B, Leutwein C, Heurtier M, Copley R, Edelmann A, Querfurth E, Rybin V, Drewes G, Raida M, Bouwmeester T, Bork P, Seraphin B, Kuster B, Neubauer G, Superti-Furga G: Functional organization of the yeast proteome by systematic analysis of protein complexes. Nature 2002, 415:141-147.

13. Gavin A, Aloy P, P G, Krause R, Boesche M, Marzioch M, Rau C, Jensen L, Bastuck S, Dmpelfeld B, Edelmann A, Heurtier M, Hoffman V, Hoefert C, Klein K, Hudak M, Michon A, Schelder M, Schirle M, Remor M, Rudi T, Hooper S, Bauer A, Bouwmeester T, Casari G, Drewes G, Neubauer G, Rick J, Kuster B, Bork P, Russell R, Superti-Furga G: Proteome survey reveals modularity of the yeast cell machinery. Nature 2006, 440:631-636.

14. Wilson K, Rocha A, Padmanabhan K, Wang K, Chen Z, Jin Y, Mihelcic J, Samatova N: Detecting pathway cross-talks by analyzing conserved functional modules across multiple phenotype-expressing organisms. Proceedings of the IEEE International Conference Bioinformatics \& Biomedicine 2011, 443-449.

15. Kastenmuller G, Schenk M, Gasteiger J, Mewes H: Uncovering metabolic pathways relevant to phenotypic traits of microbial genomes. Genome Biol 2009, 10:R28.

16. Slonim N, Elemento O, Tavazoie S: Ab initio genotype-phenotype association reveals intrinsic modularity in genetic networks. Mol Syst Biol 2006, 2:2006.0005.

17. Nath K, Das D: Improvement of fermentative hydrogen production: Various approaches. Appl Microbiol Biotechnol 2004, 65:520-529.

18. Kapdan Fl, Kargi : Bio-hydrogen production from waste materials. Enzyme Microb Technol 2006, 38:569-582.

19. Brentner L, Peccia J, Zimmerman J: Challenges in developing biohydrogen as a sustainable energy source: Implications for a research agenda. Environ Sci Technol 2010, 44:2243-2254.

20. Rey F, Heiniger E, Harwood C: Redirection of metabolism for biological hydrogen production. Appl Environ Microbiol 2007, 73:1665-1671.
21. Rey F, Oda Y, Harwood C: Regulation of uptake hydrogenase and effects of hydrogen utilization on gene expression in Rhodopseudomonas palustris. J Bacteriol 2006, 188:6143-6152.

22. Lathe W, Williams J, Mangan M, Karolchik D: Genomic data resources: Challenges and promises. Nature Edu 2008, 1(3).

23. Lopez-Gomollón S, Hernández J, Pellicer S, Angarica V, Peleato M, Fillat M: Cross-talk between iron and nitrogen regulatory networks in Anabaena (Nostoc) sp. PCC 7120: Identification of overlapping genes in FurA and NtcA regulons. J Mol Biol 2007, 374:267-281.

24. Kovacs KL, Bagi Z, Balint B, Fodor Bd, Scnadi G, Csaki R, Hanczar T, Kovacs AT, Maroti G, Perei K, Toth A, Rakhely G: Novel approaches to exploit microbial hydrogen metabolism Biohydrogen III. USA: Elsevier Inc; Miyake J, Igarashi Y, Rogner M 2004:.

25. Vignais $\mathrm{P}$, Billoud $\mathrm{B}$, Meyer J: Classification and phylogeny of hydrogenases. FEMS Microbiol Rev 2001, 25:455-501.

26. Miyake J: Biohydrogen New York, USA: Plenum Press; 1998.

27. Shima S, Thauer R: A third type of hydrogenase catalyzing $\mathrm{H} 2$ activation. Chem Rec 2007, 7:37-46.

28. White D: The physiology and biochemistry of prokaryotes Oxford, England: Oxford University Press, Inc; 2000.

29. Butland G, Zhang J, Yang W, Sheung A, Wong P, Greenbalt J, Emili A, Zamble D: Interactions of the Es-cherichia coli hydrogenase biosynthetic proteins: HybG complex formation. FEBS Lett 2006, 580:677-681.

30. Guerrini O, Soucaille P, Girbal L, Guigliarelli B, Leger C, Burlat B, Léger C: Characterization of Two 2[4Fe4S] Ferredoxins from Clostridium acetobutylicum. Curr Microbiol 2008, 56:261-267.

31. Yu J, Takahashi P: Biophotolysis-based hydrogen production by Cyanobacteria and green microalgae. In Communicating Current Research and Educational Topics and Trends in Applied Microbiology. Mendez-Vilas A 2007, 1:79-89.

32. Thomaz A, Pozzo L, Fontes A, Almeida D, Stahl C, Santos-Mallet J, Gomes S, Feder D, Ayres D, Giorgio S, Cesar C: Optical tweezers force measurements to study parasites chemotaxis. Adv Micro Tech 2009, 7367.

33. Liu Y, Li J, Sam L, Goh C, Gerstein M, Lussier Y: An integrative genomic approach to uncover molecular mechanisms of prokaryotic traits. PLOS Comput Biol 2006, 2(11):e159.

34. Gross R, Beier D: PLoS Comput Biol Caister Academic Press; 2011

35. Tamura $M, D$ 'haeseleer $P$ : Microbial genotypephenotype mapping by class association rule mining. Bioinformatics 2008, 24:1523-1529.

36. Tamura M, D'haeseler P: Comparative genome content analysis with respect to basic microbial phenotypes by class association rule mining. Proceedings of the International Conference on Systems Biology 2007.

37. Goh C, Gianoulis T, Liu J Yand Li, Paccanaro A, Lussier Y, Gerstein M: Integration of curated databases to identify genotype-phenotype associations. BMC Genomics 2006, 7:257.

38. Vitreschak AG, Mironov A, Lyubetsky V, Gelfand M: Comparative genomic analysis of T-box regulatory systems in bacteria. RNA 2008, 14:717-735.

39. Jensen L, Kuhn M, Stark M, Chaffron S, Creevey C, Muller J, Doerks T, Julien P, Roth A, Simonovic M, Bork P, von Mering C: STRING 8-a global view on proteins and their functional interactions in 630 organisms. Nucleic Acids Res 2009, 37:D412-416.

40. Tatusov R, Galperin M, Natale D, Koonin E: The COG database: A tool for genome-scale analysis of protein functions and evolution. Nucleic Acids Res 2000, 28:33-36.

41. Prelic A, Bleuler S, Zimmermann P, Wille A, Bühlmann P, Gruissem W, Hennig L, Thiele L, Zitzler E: A systematic comparison and evaluation of biclustering methods for gene expression data. Bioinformatics 2006, 22:1122-1129.

42. North B, Curtis D, Sham P: A note on the calculation of empirical $p$-values from Monte Carlo procedures. Am J Hum Genet 2002, 71:439-441.

43. Zhang B, Park B, Karpinets T, Samatova N: From pull-down data to protein interaction networks and complexes with biological relevance. Bioinformatics 2008, 24(7):979-986.

doi:10.1186/1477-5956-10-S1-S2

Cite this article as: Padmanabhan et al:: In-silico identification of phenotype-biased functional modules. Proteome Science 2012 10(Suppl 1):S2. 